\title{
Spatial prisoner's dilemma games with increasing size of the interaction neighborhood on regular lattices
}

\author{
WANG Juan ${ }^{1,2}$, XIA ChengYi ${ }^{1,2^{*}}$, WANG YiLing ${ }^{3}$, DING Shuai ${ }^{4} \&$ SUN JunQing ${ }^{1,2}$ \\ ${ }^{1}$ Tianjin Key Laboratory for Control Theory and Complicated Industry Systems, Tianjin University of Technology, Tianjin 300384, China; \\ ${ }^{2}$ Key Laboratory of Computer Vision and System (Ministry of Education), Tianjin University of Technology, Tianjin 300384, China; \\ ${ }^{3}$ School of Life Science, Shanxi Normal University, Linfen 041000, China; \\ ${ }^{4}$ Institute of Computer Network Systems, Hefei University of Technology, Hefei 230009, China
}

Received August 22, 2011; accepted October 3, 2011

\begin{abstract}
We studied the evolution of cooperation in the prisoner's dilemma game on a square lattice where the size of the interaction neighborhood is considered. Firstly, the effects of noise and the cost-to-benefit ratio on the maintenance of cooperation were investigated. The results indicate that the cooperation frequency depends on the noise and cost-to-benefit ratio: cooperation reaches a climax as noise increases, but it monotonously decreases and even vanishes with the ratio increasing. Furthermore, we investigated how the size of the interaction neighborhood affects the emergence of cooperation in detail. Our study demonstrates that cooperation is remarkably enhanced by an increase in the size of the interaction neighborhood. However, cooperation died out when the size of the interaction neighborhood became too large since the system was similar to the mean-field system. On this basis, a cluster-forming mechanism acting among cooperators was also explored, and it showed that the moderate range of the neighborhood size is beneficial for forming larger cooperative clusters. Finally, large-scale Monte Carlo simulations were carried out to visualize and interpret these phenomena explicitly.
\end{abstract}

prisoner's dilemma game, emergence of cooperation, size of interaction neighborhood

Citation: Wang J, Xia C Y, Wang Y L, et al. Spatial prisoner's dilemma games with increasing size of the interaction neighborhood on regular lattices. Chin Sci Bull, 2012, 57: 724-728, doi: 10.1007/s11434-011-4890-4

Various mechanisms of evolutionary game theory have been presented to explain the survival, sustenance and emergence of cooperation among selfish individuals in biological and social systems; e.g. kin selection [1], direct [2-4] and indirect [5,6] reciprocity, voluntary participation [7-10] and spatial extensions [11-16]. As a paradigm of pair-wise interaction games, the prisoner's dilemma game (PDG) has attracted much attention [17-21]. In the original form of the PDG, there are two behavior options: each of two players must simultaneously choose to cooperate $(C)$ or to defect (D). If both players choose $C$, they will receive the reward $R$ separately, but only the punishment $P$ for mutual defection. If two players take different strategies, then the defector will get the highest payoff of temptation $T$, while the cooperator

*Corresponding author (email: xialooking@163.com) will be left with the lowest sucker's payoff $S$. These payoffs usually satisfy the elementary payoff ranking $T>R>P>S$ and the additional required condition $(T+S)<2 R$ in repeated interactions. Henceforth, defection is the optimal choice for each player irrespective of the decision of his opponent, and defection will become widespread. Every player will end up with the payoff $P$ instead of the payoff $R$, which yields the social dilemmas as depicted in [22].

According to the motivation of the non-equilibrium kinetic Ising model in statistical physics, the PDG has been well studied for a spatially structured population in past decades [11-23]. In these models, players are distributed at the sites of different lattices or graphs, and each player can update his/her strategy and has a given probability of adopting his/her neighbor's strategies at each time step. Recently, many real systems have been found to exhibit 
complex topological characteristics that include small-world and scale-free properties, and complex networking approaches have become a powerful tool to analyze and understand complex real systems, such as those of global climate change and ecological research [24], language cluster formation in linguistic networks [25], biological interaction among proteins [26], and statistical analyses of Chinese semantic networks [27]. In addition, epidemic dynamics taking place in complex networks have received much interest in the scientific communities [28,29]. Thus, investigating cooperative dynamics in systems with complex topology has become an active topic, and the great enhancement of cooperative behavior can be attributed to heterogeneous interactions among players within real systems. For example, when the PDG on scale-free networks was considered, a dramatic improvement in cooperation was observed because competition between the cooperation and defection hubs was the main force accelerating cooperation among players [30,31]. Besides the above-mentioned topological inhomogeneity, synchronous or asynchronous updating activities, such as different teaching capabilities [32], random selections [33], and heterogeneous aspirations [34], which play an important role in promoting cooperation, have also been identified. However, systematic investigation becomes increasingly difficult for more complex topologies and various updating rules. Apart from a large number of parameters describing the payoffs, the connectivity structure and the evolution rules need to be further considered when we discuss the cooperative dynamics prevalently found in the real world.

In this paper, we discuss the effects of noise and strategy payoffs on cooperative behavior considering a PDG on a regular lattice. Notably, increasing the number of neighbors $n$ has a favorable effect on defection [35-40]. Our goal is to explore whether the effect of the size of the neighborhood $n$ is beneficial for the maintenance of cooperation. For this purpose, five neighborhoods of different size, namely the von Neumann neighborhood including only the nearest neighbors $(n=4)$, the Moore neighborhood with the nearest and second-nearest neighbors $(n=8)$, a $5 \times 5$ box excluding the player in question $(n=24)$, a $7 \times 7$ box excluding the player in question $(n=48)$ and a $9 \times 9$ box excluding the player in question $(n=80)$, are considered on a regular lattice. Figure 1 exactly shows the distribution of the interaction neighborhood. Our observations suggest that cooperation frequency $f_{\mathrm{c}}$ relies on the noise and strategy payoff. Additionally, we predict that cooperation is heavily promoted for a moderate size range of the interaction neighborhood.

\section{The game model}

In our game model, we consider the evolutionary PDG on a square lattice with a periodic boundary. Each player, occupying one of nodes on the square lattice, is a pure strategist

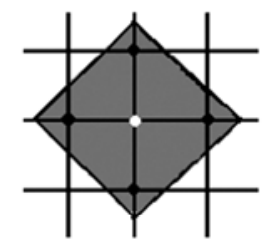

(a)

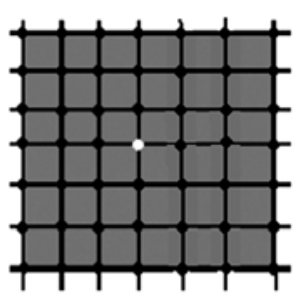

(d)

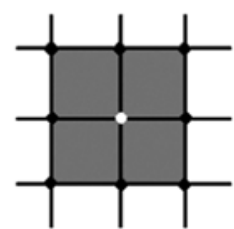

(b)

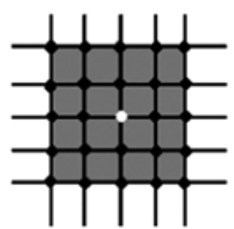

(c)
Figure 1 Examples of the number of neighbors $n$ in the PDG on regular lattices. The gray shaded areas represent the players who participate in the games at a time step. The white dots denote the focal players, whereas the black dots characterize the neighbors surrounding the focal players. From (a) to (e), the values of $n$ are 4, 8, 24, 48 and 80, respectively.

and can only follow one of two strategies: cooperation $(C)$ or defection $(D)$. In the procedure of evolution, each player is confined to interact only with his/her local neighbors and the payoff depends on his/her strategy as well as that of his/her neighbors. For simplicity and without loss of generality, we suppose that the cooperation yields a benefit $b$ to the co-player at a cost $c(b>c)$. If both players are cooperative, both gain the benefit $R=b-c$. If both choose to defect, neither is rewarded and both get the benefit $P=0$. When one player betrays the other one, the defector avoids any cost and receives benefit $T=b$, whereas the cooperator's benefit is reduced by the cost and obtains $S=-c$. Following previous studies [11,31], we adopt the rescaled payoffs depending on a single parameter; i.e. $R=1, T=1+r, S=-r$ and $P=0$, where $r=c /(b-c)$ denotes the cost-to-benefit ratio (or the ratio of the cost of cooperation to the net benefit of cooperation).

In the PDG, the payoff $P_{x}$ for a player $x$ is determined by the payoffs accumulated in his/her interaction with his/her neighbors. After each time step, player $x$ can reassess and imitate one of the more successful neighbor's strategies by comparing his/her payoff with that of a randomly selected neighbor $y$. Following previous studies [11-14,22,33-40], player $x$ can follow the strategy of a randomly selected neighbor $y$ with the probability depending on the payoff difference $\left(P_{x}-P_{y}\right)$ :

$$
W\left(s_{x} \rightarrow s_{y}\right)=\frac{1}{1+\exp \left[\left(P_{x}-P_{y}\right) / K\right]},
$$

where the parameter $K$ denotes the amplitude of noise in the strategy adoption. In the limit of $K$, the strategy of neighbor $y$ is always adopted if $P_{y}>P_{x}$. In the case of $K \rightarrow \infty$, player $x$ switching to the strategy of neighbor $y$ is a stochastic selection. In this work, we focus on the case of $K>0$. 
In this model, Monte Carlo simulations are performed for a population consisting of $N=100 \times 100$ individuals. Each individual is initially assigned with equal probability $(50 \%)$ of randomly selecting one of the two strategies: $C$ or $D$. In what follows, we mainly study the average frequency of cooperators $f_{\mathrm{c}}$ when the system is in a stationary state. The key quantity $f_{\mathrm{c}}$ is measured by averaging over 20 realizations, each of which is an average of the last 50000 of the 60000 Monte Carlo time steps.

\section{Numerical results and discussion}

We start by analyzing the effects of the amplitude of noise and the strategy payoff on the frequency of cooperators. For simplicity of expression, we use the variation in parameter $r$ to represent the variation in strategy payoff. Figure 2 demonstrates clearly that when the variation in noise is considered, the trend of the variation in cooperator's frequency $f_{\mathrm{c}}$ is similar irrespective of the value of $r$. As noise $K$ increases, the cooperator's frequency $f_{\mathrm{c}}$ increases monotonously until reaching a maximum value; i.e. there is a threshold $K_{\mathrm{s}}$ for the optimum composition of cooperation, which is analogous to the so-called coherence resonance [19]. Naturally, for $K>K_{\mathrm{s}}$, the cooperator's frequency $f_{\mathrm{c}}$ decreases evidently and even vanishes. This means that cooperation is affected by the amplitude of the noise $K$. However, under the same noise level, how does the cooperator's frequency $f_{\mathrm{c}}$ change with the parameter $r$ ? From Figure 2, we find that the smaller that $r$ is, the larger $f_{\mathrm{c}}$ will be. In the case of $r=0$, because the value of $T$ equals that of $R$, the temptation to defect is reduced severely, and the probabilities of choosing $C$ or $D$ are nearly equal. For $r>0$, it is well known that defection is more beneficial, and cooperation is seriously inhibited. It is more interesting that within the special region of $K$, cooperators cannot remain alive if the value of $r$ increases; i.e. the system will convert from a state of co-existence of cooperators and defectors to an absorbing state of only defectors. This phenomenon reveals a common view that a decrease in the cost-to-benefit ratio (i.e. temptation to defection) promotes cooperation greatly. Thus, appropriate values of $K$ and $r$ favor the sustenance of cooperation to some extent.

Next, we turn to the role of the size of the interaction neighborhood $n$ in promoting cooperation. In this case, our model can be considered a more accurate extension of the model of Szabó [38] where $w=1$ for players of type B. Figure 3 shows the effect of $n$ on the cooperator's frequency $f_{\mathrm{c}}$ by varying $K$ while keeping $r$ fixed. It is clear that except in the case of $n=80$, the frequency of cooperators $f_{\mathrm{c}}$ increases non-monotonously with the continuous increase in $K$; that is, increasing $K$ results in a climax of $f_{\mathrm{c}}$ for other types of neighborhood, which is in accordance with the phenomenon in Figure 2.

More importantly, it is clearly seen that $f_{\mathrm{c}}$ remarkably

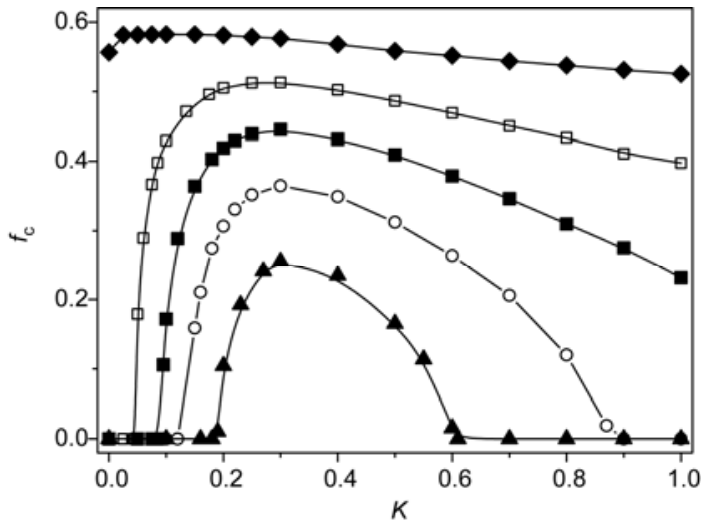

Figure 2 Frequency of cooperators $f_{\mathrm{c}}$ as a function of the amplitude of noise $K$ for different values of $r(r=0,0.01,0.02,0.03$ and 0.04 from top to bottom). The simulations are performed on a regular lattice with the number of interaction neighbors $n=4$.

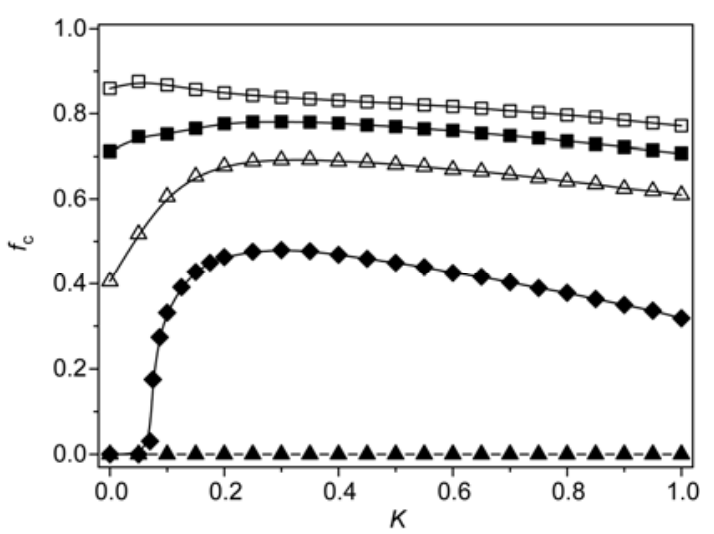

Figure 3 Frequency of cooperators $f_{\mathrm{c}}$ as a function of the amplitude of noise $K$ for $r=0.015$ on a square lattice. The simulation results are for different values of $n$ ( $n=48,24,8,4$ and 80 from top to bottom).

increases and cooperators ultimately dominate the games if $n$ increases for mid-range values of $n=24$ and 48. Apparently, for $n=4$, cooperators become extinct if the value of $K$ is low enough. In the case of $n=8$, the robustness of cooperation is slightly weaker than in the cases of $n=24$ and 48 , but better than that in the case of $n=4$. When the size of the interaction neighborhood is sufficiently large (i.e. $n=80$ ), the result is consistent with mean-field-type behavior and the extinction of cooperation is thus inevitable. Consequently, these results show that the cooperator's frequency $f_{\mathrm{c}}$ does not monotonously depend on the size of the neighborhood, which assumes an important role in the emergence of cooperation. Cooperation can be better promoted under the condition of a moderate size-range of the interaction neighborhood, but is inhibited in the sufficiently large case.

To investigate the procedure of cooperation sustenance in detail, we plot $f_{\mathrm{c}}$ versus $r$ for different sizes of neighborhood $n$ in Figure 4. On one hand, we see that as the cost-to-benefit ratio (i.e. the temptation to defect) $r$ increases, $f_{\mathrm{c}}$ decreases and even vanishes as depicted in Figure 2. On the other hand, it is clear that $f_{\mathrm{c}}$ depends on $n$. We find that in the cases of $n=80$ and 48, although cooperators absolutely govern 


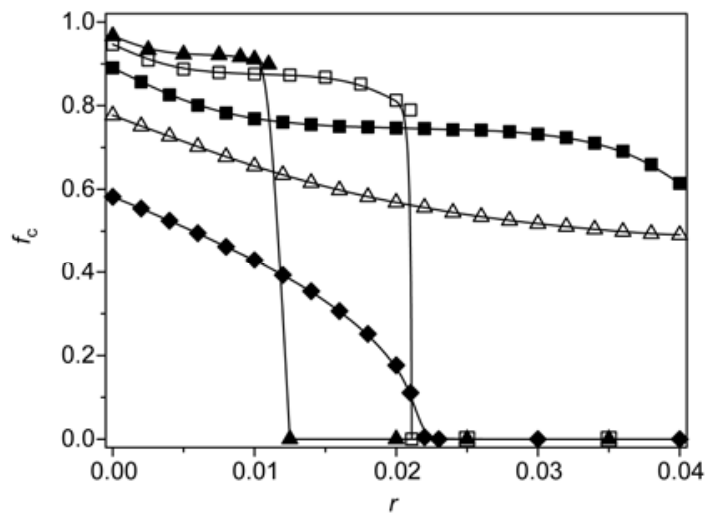

Figure 4 Frequency of cooperators $f_{\mathrm{c}}$ as a function of the cost-to-benefit ratio $r$ for $K=0.1$ on square lattices. The simulation results are obtained for different values of $n$ (solid triangles, hollow triangles, hollow squares, solid squares and solid diamonds represent results for $n=80,8,48,24$ and 4).

the games at first, they inevitably tend to die out as $r$ reaches larger values, where defection becomes influential. When the focal player has a moderate number of neighbors (i.e. $n=24)$, it becomes instantly obvious that mutual cooperation is more successful in obtaining a higher profit irrespective of the value of $r$. Choosing defection results in the scene that if most neighbors of the focal player also choose defection, which results in clusters of defectors because of the many interaction neighbors, his/her expected payoff will be much less than that of the cooperators. Evidently, cooperation is the best strategy in such a situation. With the value of $n$ decreasing monotonously, cooperation becomes increasingly difficult and there is even a saturation state of global defectors $(n=4)$. The reason for this kind of cooperation deterioration is that the cluster-forming of defectors becomes relatively easy, which results in the smaller loss of defectors. Thus, we argue that it is a valuable observation that the maintenance of cooperation is supported remarkably by a mid-sized interaction neighborhood.

It is notable that cooperators are able to survive by forming clusters that minimize exploitation by invading defectors in spatial games $[11,13]$. A cooperator cluster consists of many cooperators that are fully conjoint. Along the boundary of these clusters, cooperators can make up their loss exploited by defectors by choosing cooperation as a member of the cooperative clusters. Here, to confirm the effect of the size of the interaction neighborhood $n$ on the cooperation explicitly, we present typical snapshots of the strategy distribution on the square lattice for different values of $n$. Figure 5 shows that the clusters of cooperators become larger as $n$ appropriately increases, while they cannot survive for a sufficiently large neighborhood.

Interestingly, we find that cooperative clusters form within a small area to minimize exploitation by defectors (Figure 5(a)). Since the size of the interaction neighborhood affects the interplay between cooperators and defectors, this further affects their payoffs. In the case of a small neighborhood, the payoff of a cooperator's clusters does not sufficiently

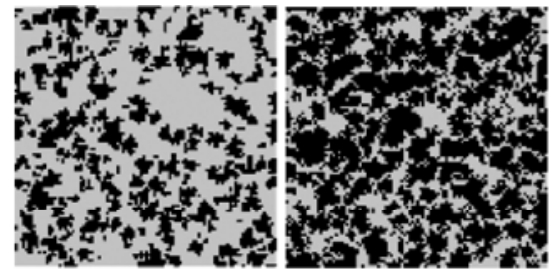

(a) (b)

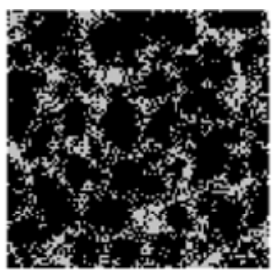

(c)

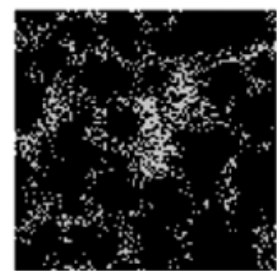

(d)

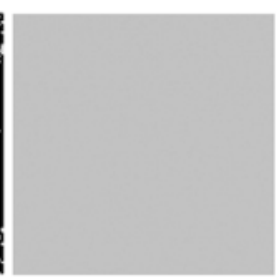

(e)
Figure 5 Five typical snapshots of the distribution of the strategies $C$ (black) and $D$ (gray) on a $100 \times 100$ regular lattice. The parameters are $K=$ 0.1 and $r=0.015$ for different values of $n$ (from (a) to (e), $n=4,8,24,48$ and 80 respectively).

exceed that of defectors, which restricts the infection and spreading of cooperation strategy due to the limited neighborhood size. However, this situation is reversed for increasing interaction sizes. Therefore, more cooperators spontaneously form clusters on the lattice for a more profitable outcome; i.e. small cooperator clusters do not receive an optimal payoff. This is more apparent in the case of midrange $n$ values (Figure 5(c) and (d)). Meanwhile, our results suggest that if the value of $n$ is moderate, defectors are highly dispersive and surround the boundary of the cooperator's clusters, which may be similar to realistic results for human society. Of course, if the size of the interaction neighborhood is large enough, the situation is similar to the mean-field prediction in which cooperation does not have survival space (Figure 5(e)).

The reason why the moderate size of the interaction neighborhood sustains cooperation is now easily understood. Cooperators among cooperative clusters usually have a higher payoff than defectors. With the size of the neighborhood increasing little by little, the dominant payoff for cooperators within the cluster becomes more visible; hence, cooperation is greatly facilitated.

\section{Conclusions}

We investigated the general question of cooperation evolution in the PDG with different sizes of interaction neighborhoods on regular lattices. Beginning with the variation in noise $K$ and cost-to-benefit ratio $r$, we showed that the frequency of cooperators $f_{\mathrm{c}}$ relies on the values of these parameters and varies quantitatively with them. When increasing the noise, we observed that the peaks of $f_{\mathrm{c}}$ are independent of the value of $r$. At the same time, we demonstrated that an increase in $r$ is not beneficial for the promotion and sustenance of cooperation. Thus, the noise $K$ and cost-to-benefit ratio $r$ 
play an indispensable role in the evolution of cooperation. In addition, we mainly analyzed the effect of the size of neighborhood $n$ on the emergence and sustenance of cooperation. We found that the largest increase in cooperation is obtained for a moderately sized interaction neighborhood, which confirms our conjecture and differs from previous results. More interestingly, in the procedure of evolution, cooperators form compact clusters to survive and these clusters become larger as $n$ increases little by little, which can protect the cooperators from being exploited by defectors. Consequently, the size of the interaction neighborhood is important in maintaining cooperation, and provides new views and explanations of some social phenomena.

In light of the above conclusions, many further investigations need to be carried out. Investigations need to ascertain whether the results obtained in this work also apply to the snowdrift game on a lattice, and how the results vary if other non-regular lattices are introduced. Additionally, the greatest issues of concern relate to the individual payoffs in these social dilemmas, such as how the payoffs vary in detail with a change in the parameter $n$.

This work was supported by the National Natural Science Foundation of China (60904063 and 70871090), the Tianjin Municipal Natural Science Foundation (11JCYBJC06600 and 11ZCKFGX00900) and the Research Fund of Tianjin University of Technology (LGYM200918).

1 Hamilton W D. The evolution of social behavior. J Theor Biol, 1964, 7: $1-16$

2 Nowak M A, Sigmund K. Evolution of indirect reciprocity by image scoring. Nature, 1998, 393: 573-577

3 Axelrod R, Hamilton W D. The evolution of cooperation. Science, 1981, 211: 1390-1396

4 Wang Z, Du W B, Cao X B, et al. Integrating neighborhoods in the evaluation of fitness promotes cooperation in the spatial prisoner's dilemma game. Physica A, 2011, 390: 1234-1240

5 Fehr E, Gachter S. Altruistic punishment in humans. Nature, 2002, 415: $137-140$

6 Nowak M A, Sigmund K. Evolution of indirect reciprocity. Nature, 2005, 437: 1291-1298

7 Rong Z H, Wu Z X. Effect of the degree correlation in public goods game on scale-free networks. Europhys Lett, 2009, 87: 30001

8 Hauert C, De Monte S, Hofbauer J, et al. Volunteering as red queen mechanism for cooperation in public goods game. Science, 2002, 296: $1129-1132$

9 Semmann D, Krambeck H J, Milinski M. Volunteering leads to rock-paper-scissors dynamics in a public goods game. Nature, 2003, 425: 390-393

10 Wang Z, Xu Z J, Zhang L Z. Maintenance of cooperation induced by punishment in public goods games. Chin Phys B, 2009, 19: 110201

11 Szabó G, Fáth G. Evolutionary games on graphs. Phys Rep, 2007, 446: $97-216$

12 Cao X B, Du W B, Rong Z H. Evolutionary public goods game on scale-free networks with heterogeneous investment. Physica A, 2010, 389: $1273-1280$

13 Wang $\mathrm{Z}$, Perc M. Aspiring to the fittest and promotion of cooperation in the prisoner's dilemma game. Phys Rev E, 2010, 82: 021115

14 Xia C Y, Zhao J, Wang J, et al. Influence of vertex weight on cooperative behavior in a spatial snowdrift game. Phys Scr, 2011, 84:
025802

15 Du W B, Cao X B, Yang H X, et al. Evolutionary prisoner's dilemma on Newman-Watts social networks with an asymmetric payoff distribution mechanism. Chin Phys B, 2010, 19: 010204

16 Wang Z, Xu Z J, Zhang L Z. Punishment in optional public goods games. Chin Phys B, 2009, 19: 100204

17 Doebeli M, Hauert C. Models of cooperation based on the prisoner's dilemma and the snowdrift game. Ecol Lett, 2005, 8: 748-766

18 Wang Z, Perc M. Aspiring to the fittest and promotion of cooperation in the prisoner's dilemma. Phys Rev E, 2010, 82: 021115

19 Perc M. Coherence resonance in a spatial prisoner's dilemma game. New J Phys, 2006, 8: 22

20 Nowak M A, Bonhoeffer S, May R M. A simple rule for the evolution of cooperation on graphs. Nature, 2006, 441: 502-505

21 Du W B, Cao X B, Hu M B. The effect of asymmetric payoff mechanism on evolutionary networked prisoner's dilemma game. Physica A, 2009, 388: 5005-5012

22 Hardin G. Extensions of "The tragedy of the commons". Science, 1998, 280: 682-683

23 Chen Y, Qin S M, Yu L C, et al. Emergence of synchronization induced by the interplay between two prisoner's dilemma games with volunteering in small-world networks. Phys Rev E, 2008, 77: 032103

24 Liu Z L, Peng C H, Xiang W H, et al. Application of artificial neural networks in global climate change and ecological research: An overview. Chin Sci Bull, 2010, 55: 3853-3863

25 Liu H T, Li W W. Language clusters based on linguistic complex networks. Chin Sci Bull, 2010, 55: 3458-3465

26 Chang S, Gong X Q, Jiao X, et al. Network analysis of protein-protein interaction. Chin Sci Bull, 2010, 55: 814-822

27 Liu H T. Statistical properties of Chinese semantic networks. Chin Sci Bull, 2009, 54: 2781-2785

28 Liu T, Li X, Liu X P. Integration of small world networks with multi-agent systems for simulating epidemic spatiotemporal transmission. Chin Sci Bull, 2010, 55: 1285-1293

29 Xia C Y, Sun S W, Liu Z X, et al. Epidemics of SIRS model with nonuniform transmission on scale-free networks. Int J Mod Phys B, 2009, 23: 2203-2213

30 Santos F C, Pacheco J M. Scale-free networks provide a unifying framework for the emergence of cooperation. Phys Rev Lett, 2005, 95: 098104

31 Wu Z X, Rong Z H, Holme P. Diversity of reproduction time scale promotes cooperation in spatial prisoner's dilemma games. Phys Rev E, 2009, 80: 036106

32 Szolnoki A, Szabó G. Cooperation enhanced by inhomogeneous activity of teaching for evolutionary prisoner's dilemma games. Europhys Lett, 2007, 77: 30004

33 Chen X J, Wang L. Cooperation enhanced by moderate tolerance ranges in myopically selective interactions. Phys Rev E, 2009, 80: 046109

34 Perc M, Wang Z. Heterogeneous aspirations promote cooperation in the prisoner's dilemma game. PLoS ONE, 2010, 5: e15117

35 Szabó G, Vukov J, Szolnoki A. Phase diagrams for an evolutionary prisoner's dilemma game on two-dimensional lattices. Phys Rev E, 2005, 72: 047107

36 Zhang H F, Liu R R, Wang Z, et al. Aspiration-induced reconnection in spatial public goods game. Eur Phys Lett, 2011, 94: 18006

37 Wang Z, Murks A, Du W B, et al. Coveting the neighbors fitness as a means to resolve social dilemmas. J Theor Biol, 2011, 277: 19-26

38 Szabó G, Szolnoki A. Cooperation in spatial prisoner's dilemma games with two types of players for increasing number of neighbors. Phys Rev E, 2009, 79: 016106

39 Xu Z J, Wang Z, Zhang L Z. Bounded rationality in volunteering public goods games. J Theor Biol, 2010, 264: 19-23

40 Szolnoki A, Perc M, Szabó G. Phase diagrams for three-strategy evolutionary prisoner's dilemma games on regular graphs. Phys Rev E, 2009, 80: 056104

Open Access This article is distributed under the terms of the Creative Commons Attribution License which permits any use, distribution, and reproduction in any medium, provided the original author(s) and source are credited. 